\title{
High-power low-frequency current oscillations in germanium samples
}

\author{
S.P. Pavljuk \\ Department of Radiphysics, National Taras Shevchenko University of Kyiv, acad. Glushkov Ave., 2, build. 5, Kyiv 03127, Ukraine \\ Phone: +38(044) 266 6347; e-mail: pps@univ.kiev.ua
}

\begin{abstract}
Experimental investigation of high-power low-frequency current oscillations in germanium samples with low injecting contacts is discussed in this article. The results obtained were explained by periodic formation, transport along the sample, and collapse of the thermal gradient-drift (TGD) domain. The domain formation mechanism is considered in details in [1].
\end{abstract}

Keywords: current, oscillation, temperature, domain

Paper received 24.12.02; accepted for publication 18.03.03.

Current oscillations in germanium samples, similar to those observed in this paper, were presented earlier in [2], but the mechanism of their appearance was not discussed there. It is only known that experiments were performed on the samples with contacts having different injecting ability. It was experimentally shown, that only one current peak was invariably observed in a germanium sample with two high injecting contacts ( $p$ - $n$-junctions), next the sample cracked. Periodic current oscillations were observed in the samples with both tin contacts possessing low injecting ability.

Reasons related to special features of the current flow in samples with contacts having different injecting ability become clear, following the examination of the equation describing the behavior of electron-hole plasma in semiconductor with concentrations of electrons $n$ and holes $p(p \approx n)$

$$
\frac{\partial \Delta p}{\partial t}=D \frac{\partial^{2} \Delta p}{\partial x^{2}}-\mu E \frac{\partial \Delta p}{\partial x}-\frac{\Delta p}{\tau}+A \frac{1}{T} \frac{d T}{d x},
$$

where: $\Delta p=p-p_{0}$ is the plasma concentration variation, $D$ and $\mu$ are the effective diffusion coefficient of electronhole pairs and their effective mobility, respectively, $E$ is the electric field strength in semiconductor, $\tau$ is the effec- tive pair lifetime, $A$ is the factor dependent on the current value and semiconductor material, and $T$ is the temperature.

The first term in the right-hand side of the equation is associated with the charge carrier diffusion along the sample, the second - with their drift in the electric field $E$, the third - with the generation-recombination process in semiconductor, while the fourth - with $b$-drift [3].

Let us consider how the relationship between the terms in the right-hand side of the equation reflects the physical processes in a germanium sample.

The thermal gradient-drift (TGD) domain in germanium moves towards the negative contact from the place of its emergence [2]. If the injection coefficient of this contact is so high that the diffusion term becomes greater than the term associated with $b$-drift, then the TGD domain will be unable to reach this contact. The fact is that the TGD domain comes to a stop when it reaches the diffusion area near the negative contact. It takes place because of $b$-drift that could not extract charged carriers from the diffusion area which pass there from the contact due to injection. These carriers are accumulated in the diffusion area and destroy the domain. Moreover, the area of the TGD domain location is strongly heated with the flowing current, and the temperature gradient de- 


\section{S.P. Pavljuk: High-power low-frequency current oscillations in ...}

creases in the domain area. At the same time, the highintensity thermal generation of electron-hole pairs takes place in the domain area. They "flood" this area and decrease its resistivity. Under the action of these two processes the TGD domain collapses, the current through the sample sharply increases, and the latter cracks [1]. Thus, only a single current peak (rather than periodic current oscillations) can be observed in such samples as we observed in our experiment.

If the injection coefficient of the negative contact is so low that the diffusion term is less than the term associated with $b$-drift, then the TGD domain is able to attain the negative contact and to disappear there without distructing the sample. Next, the cycle is repeated. These arguments were verified by experiment.

The experiments were performed both at liquid nitrogen and room temperatures. The selection of experimental conditions was based on the possible observation of different mechanisms of the TGD domain appearance and disappearance at various temperatures.

The $n$-type germanium samples with donor concentration of approximately $10^{13} \mathrm{~cm}^{-3}$ were used to observe the current oscillations in a sample dipping into the liquid nitrogen. The sample $p^{+}$-contact with high injecting ability was manufactured by indium fusing; $n$-contact with low injecting ability was soldered on by tin.

Single rectangular voltage pulses with controlled duration were applied across samples; hereat the corresponding current pulses flowing through the sample were observed (Fig. 1). At different time instants of the voltage

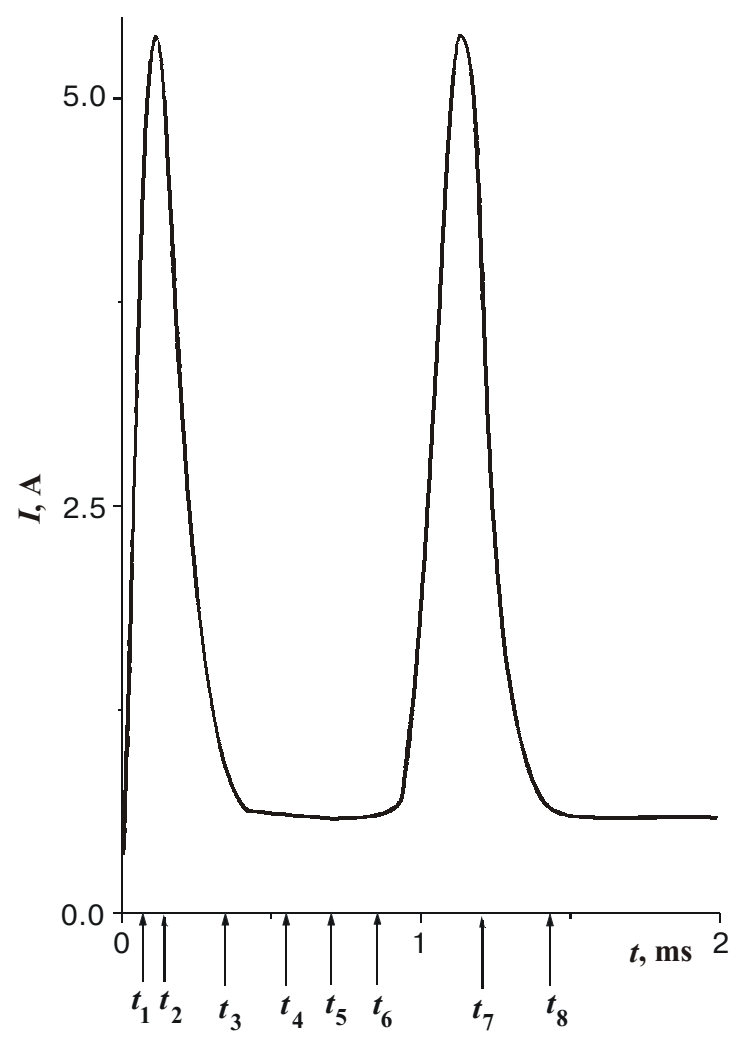

Fig. 1. Oscillogram current oscillation at the liquid nitrogen temperature. pulse shown in Fig. 1 as $t_{1} \ldots t_{8}$, the electric field distribution along the sample was determined (Fig. 2). Numbers near the curves in Fig. 2 correspond to those times.

As can be seen from these figures, at time $t_{1}$, the highest electric field strength area is located near the $n$-contact. Under this field, electrones are injected from the contact into the sample bulk, while holes are injected from the $p^{+}$-contact. As a result, the current increases up to the value sufficient for the non-uniform heating of the sample, especially of its middle part (time $t_{2}$ in Fig. 1), and for the beginning of the TGD domain formation (curve 2 in Fig. 2). The domain formation results in the sample current reduction down to its lowest value (time $t_{3}$ in Fig. 1). After its formation, the domain begins to shift from the $p^{+}$-contact towards the $n$-contact (curves 3, 4, 5, 6 in Fig. 2, respectively). At time $t_{6}$ the TGD domain reaches the $n$-contact and disappears there (curve 6 in Fig. 2). Then, the initial state is realized. The current through the sample begins to increase again (time $t_{7}$ ), a new TGD domain is formed (time $t_{8}$, Fig. 1), and the cycle repeats (curves 7 and 8 in Fig. 2, respectively).

Current oscillation will be stable, if the integral temperature of the sample does not fluctuate significantly during the current flowing. Just for the provision of such conditions, the experiments were performed in the liquid nitrogen used as coolant. It was established that the oscillation frequency is determined by the TGD domain

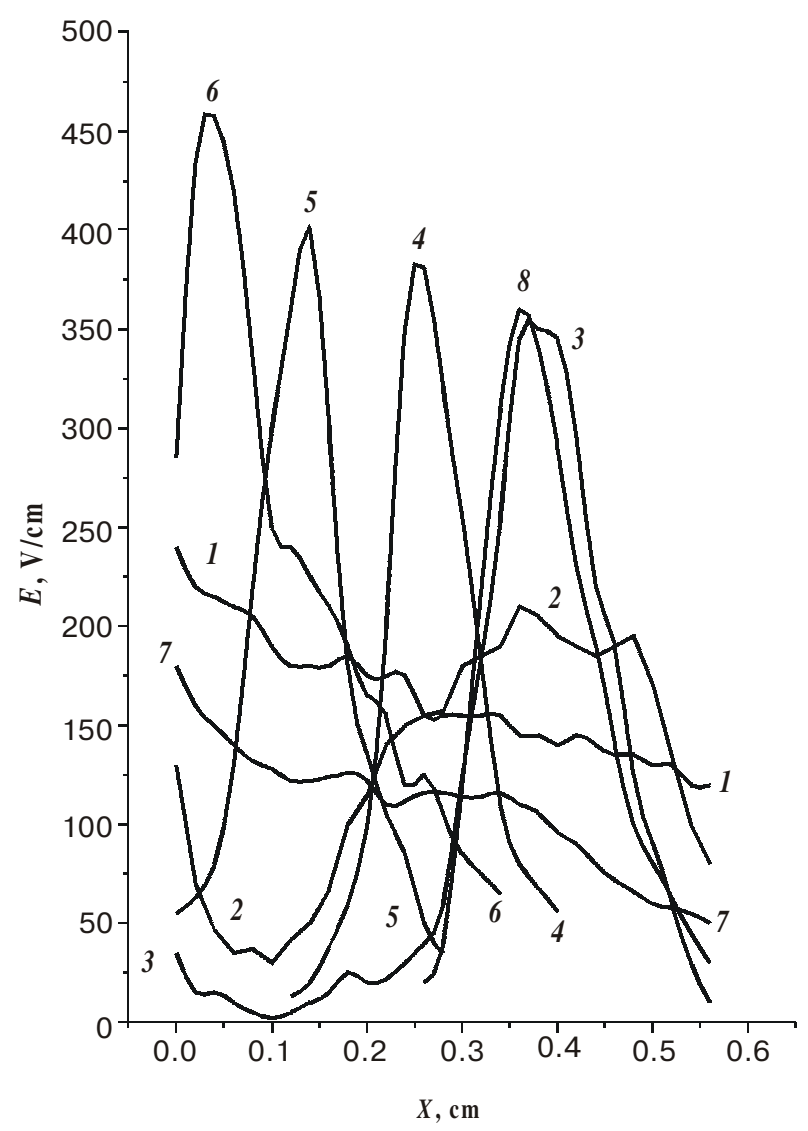

Fig. 2. Electric field distribution along the sample at corresponding time instants shown in Fig. 1. 


\section{S.P. Pavljuk: High-power low-frequency current oscillations in ...}

shift speed along the sample and the length of the latter. At sample length of $0.56 \mathrm{~cm}$ the oscillation frequency varied within the range of $1 . .5 \mathrm{kHz}$ depending on the applied voltage value.

At the voltage pulse duration less than $1 \mathrm{sec}$, current oscillations were sufficiently stable. As the pulse duration increases ( $1 \mathrm{sec}$ or more), the maximum $I_{\max }$ and minimum $I_{\min }$ current values were gradually increased with time after the pulse beginning, and the oscillation frequency also increased. When the integrated heating of the sample reached the level of the liquid nitrogen boiling, a gaseous nitrogen layer was formed around the sample, the heat sinking sharply decreased, and the sample cracked $[1,3]$.

Oscillation frequency could be changed by the variation of both the applied voltage and sample length. The length decrease from $0.56 \mathrm{~cm}$ to $0.3 \mathrm{~cm}$ increased the oscillation frequency from $1 . .5 \mathrm{kHz}$ up to $3 \ldots 10 \mathrm{kHz}$, but their stability was lowered.

The applied voltage increase resulted in the growth of $I_{\max }$ and $I_{\min }$ values, the decrease of current modulation index $K=I_{\max } / I_{\min }$, the increase of oscillation frequency and their instability. The power of such oscillations reached $500 \mathrm{~W}$.

At room temperature, investigations were performed on the germanium samples with the intrinsic conductivity (donor concentration $\approx 10^{12} \mathrm{~cm}^{-3}$ ). Both contacts were soldered on with tin and featured low injecting ability. The sample length was $0.3 \ldots 0.8 \mathrm{~cm}$, their cross-section was $0.1 \ldots 0.25 \mathrm{~cm}^{2}$. For more effective cooling, the sample was pressed to the copper heat-sink through the thermal conducting paste and thin dielectric layer.

As in the previous case, single rectangular pulses of voltage with controlled duration and amplitude were applied across the samples, and the corresponding current pulses were observed (Fig. 3). At the characteristic times $t_{1} \ldots t_{8}$, the electric field strength distribution $E(x)$ was measured along the sample (Fig. 4). The oscillograms show that current value after the voltage pulse beginning is low, and for a considerably long period (up to $4 \mathrm{~ms}$ ) increases slowly. Electric field at time $t_{1}$ is distributed along the middle part of the sample near-uniformly, and its strength is higher only near the negative contact (curve 1, Fig. 4). During this time the negative contact is heated, the temperature gradient running into $100 \mathrm{~K} / \mathrm{cm}$ occurs near the contact. In accordance with [3], the $b$ drift enriches this contact area with the non-equilibrium charged carriers increasing the current flowing through the sample. Electric field decreases near the negative contact; flowing current begins to heat the middle part of the sample, the temperature gradient of the sign required to the TGD domain formation occurs there. Carrier concentration becomes of the same order of donor concentration $10^{12} \mathrm{~cm}^{-3}$, and flowing current reduces by the factor of 100. According to [1], the TGD domain begins to shift towards the negative contact (time instants $2,3,4,5$ in Fig. 4). But low current (of the order of $0.1 \ldots 0.2 \mathrm{~A}$ ) flowing through the sample is insufficient for its non-uniform heating, proper temperature gradient maintenance,

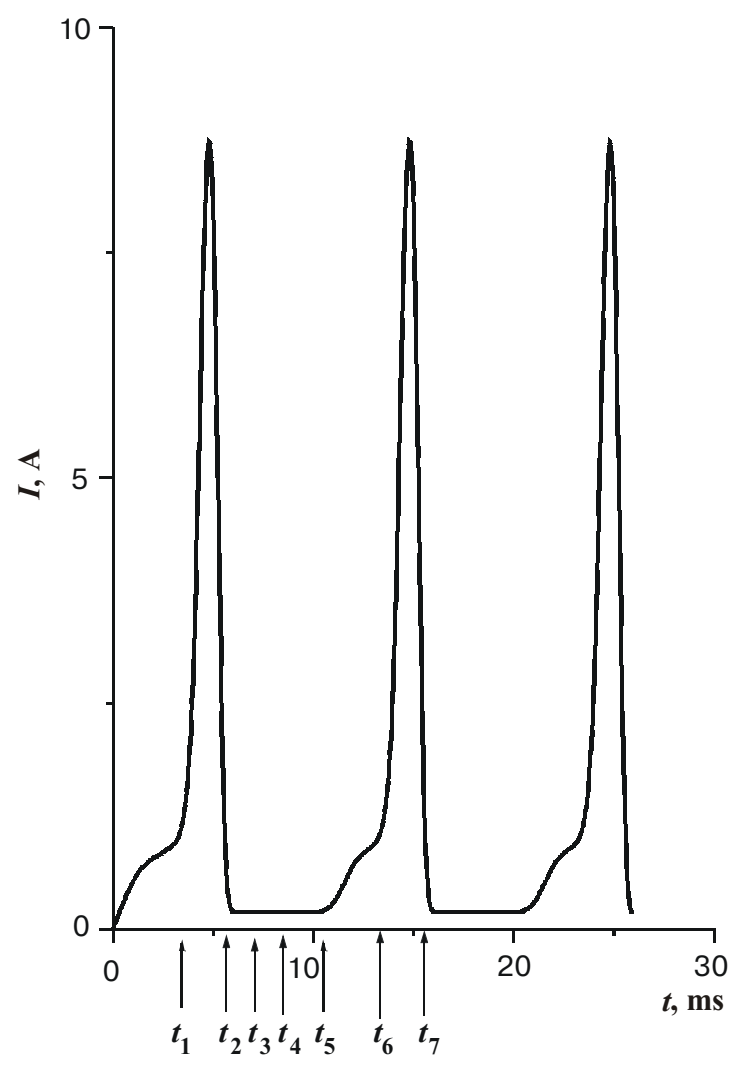

Fig. 3. Oscillogram current oscillation at the room temperature.

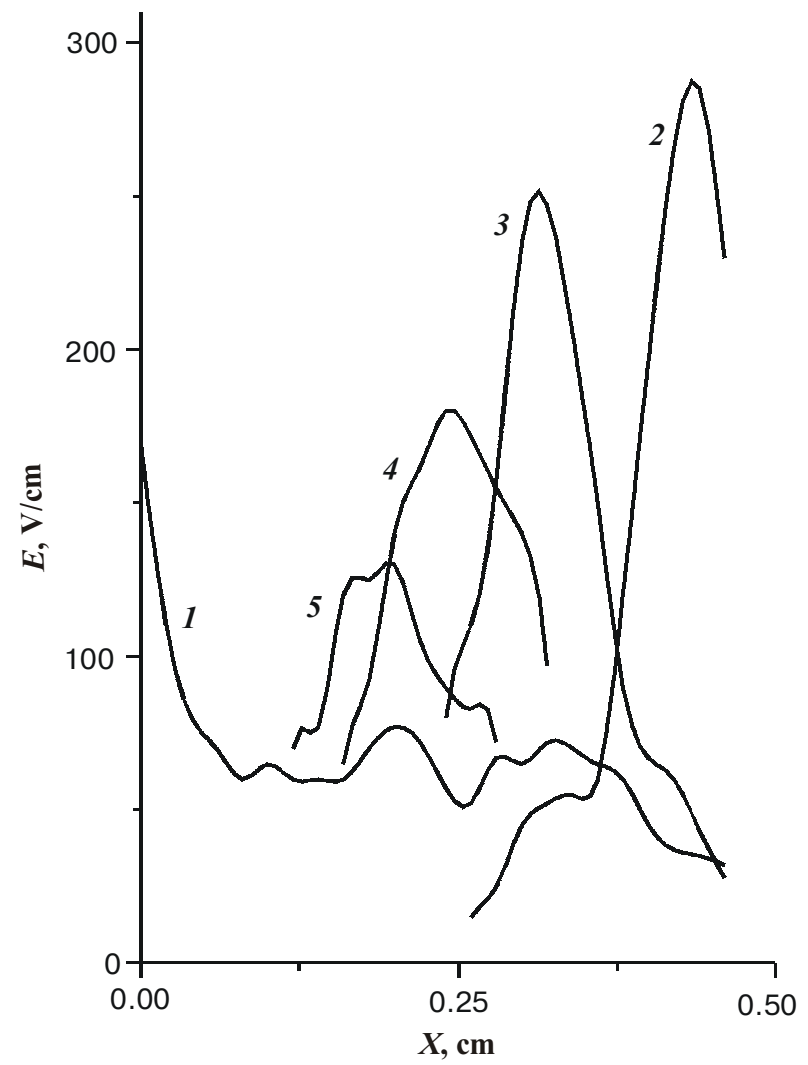

Fig. 4. Electric field distribution along the sample at corresponding time instants shown in Fig. 3. 


\section{S.P. Pavljuk: High-power low-frequency current oscillations in ...}

and the TGD domain existence. After its formation, the TGD domain disappears gradually during its shifting along the sample, carriers concentration increases inside its area, and at the moment $t_{6}$ electric field distribution follows itself at the moment $t_{1}$. A new period of oscillation begins.

Current oscillations are quite powerful running into $100 \mathrm{~W}$. Effective heat-sink maintains the oscillation parameters stability at pulse duration up to one second. Oscillation stability duration can be increased by using more effective heat-sink like diamond or sapphire.

Proceed from the results obtained, the next model of the current oscillation occurrence was proposed.

Under the action of the applied voltage pulse, the current through the sample begins to increase, the essential non-uniform heating of the sample takes place, and the TGD domain occurs. This domain occurrance reduces the current flowing through the sample by a factor of 10 or more. In such a manner, the current peak is formed.
After its formation, the domain heating begins, the temperature gradient reduces shifting the TGD domain towards the maximum gradient area. Domain is formed near the positive contact in germanium, moves along the sample, and disappears in the negative contact or near it.

The TGD domain disappearance results in current increasing and new current peak occuring. Then the cycle repeats.

\section{References}

1. V.N. Dobrovol'sky, S.P. Pavljuk. Thermal gradient-drift domain in the electron-hole plasma of semiconductors // Semiconductors 15 (1), p. 120-129 (1981), in Russian.

2. V.N. Dobrovolskii, M.N. Vinoslavskii. High Fild Domain in Electron-Hole Plasma of Germanium (II) // Phys. Stat Sol. (a) 24, p. 653-660 (1974).

3. V.N. Dobrovol'sky, S.P. Pavljuk. Drift of the electron-hole plasma caused by charge carrier mobility gradients // Semiconductors 11 (7), p. 1377-1380 (1977), in Russian. 